\title{
Papers
}

\section{A strategy to reduce cardiovascular disease by more than $80 \%$}

N J Wald, M R Law

\begin{abstract}
Objectives To determine the combination of drugs and vitamins, and their doses, for use in a single daily pill to achieve a large effect in preventing cardiovascular disease with minimal adverse effects. The strategy was to simultaneously reduce four cardiovascular risk factors (low density lipoprotein cholesterol, blood pressure, serum homocysteine, and platelet function) regardless of pretreatment levels. Design We quantified the efficacy and adverse effects of the proposed formulation from published meta-analyses of randomised trials and cohort studies and a meta-analysis of 15 trials of low dose (50-125 $\mathrm{mg}$ /day) aspirin.
\end{abstract}

Outcome measures Proportional reduction in ischaemic heart disease (IHD) events and strokes; life years gained; and prevalence of adverse effects. Results The formulation which met our objectives was: a statin (for example, atorvastatin (daily dose $10 \mathrm{mg}$ ) or simvastatin $(40 \mathrm{mg})$ ); three blood pressure lowering drugs (for example, a thiazide, a $\beta$ blocker, and an angiotensin converting enzyme inhibitor), each at half standard dose; folic acid $(0.8 \mathrm{mg})$; and aspirin $(75 \mathrm{mg})$. We estimate that the combination (which we call the Polypill) reduces IHD events by $88 \%$ (95\% confidence interval $84 \%$ to $91 \%)$ and stroke by $80 \%(71 \%$ to $87 \%)$. One third of people taking this pill from age 55 would benefit, gaining on average about 11 years of life free from an IHD event or stroke. Summing the adverse effects of the components observed in randomised trials shows that the Polypill would cause symptoms in $8-15 \%$ of people (depending on the precise formulation). Conclusion The Polypill strategy could largely prevent heart attacks and stroke if taken by everyone aged 55 and older and everyone with existing cardiovascular disease. It would be acceptably safe and with widespread use would have a greater impact on the prevention of disease in the Western world than any other single intervention.

\section{Introduction}

Heart attacks, stroke, and other preventable cardiovascular diseases kill or seriously affect half the population of Britain. Western diet and lifestyle have increased the population levels of several of the causal "risk factors," and their combined effects have made the diseases common. Cardiovascular disease can be avoided or delayed, but the necessary changes to Western diet and lifestyle are not practicable in the short term. Randomised trials show that drugs to lower three risk factors-low density lipoprotein (LDL) cholesterol, ${ }^{1}$ blood pressure, ${ }^{2-6}$ and platelet function (with aspirin $)^{7}$-reduce the incidence of ischaemic heart disease (IHD) events and stroke. Evidence that lowering serum homocysteine (with folic acid) reduces the risk of these diseases is largely observational but still compelling. ${ }^{9}{ }^{10}$

Drug treatment to prevent IHD events and stroke has generally been limited to single risk factors, to targeting the minority of patients with values in the tail of the risk factor distribution, and to reducing the risk factors to "average" population values. This policy can achieve only modest reductions in disease. ${ }^{11}$ A large preventive effect would require intervention in everyone at increased risk irrespective of the risk factor levels; intervention on several reversible causal risk factors together; and reducing these risk factors by as much as possible. ${ }^{11}$

We describe a strategy to prevent cardiovascular disease based on these three principles ${ }^{12}$ and quantify the overall preventive effect. We show that a daily treatment, the Polypill, comprising six components, each lowering one of the above four risk factors, would prevent more than $80 \%$ of IHD events and strokes, with a low risk of adverse effects. This strategy would be suitable for people with known cardiovascular disease and for everyone over a specified age (say 55), without requiring risk factors to be measured.

\section{Methods}

We identified categories of drugs or vitamins used to modify LDL cholesterol, blood pressure, homocysteine, and platelet function. For LDL cholesterol, statins are the drugs of choice. ${ }^{13}{ }^{14}$ For lowering blood pressure, we considered all five main categories of drugs: thiazides, $\beta$ blockers, angiotensin converting enzyme (ACE) inhibitors, angiotensin II receptor antagonists, and calcium channel blockers. ${ }^{13}$ Serum homocysteine is most effectively reduced by folic acid; vitamins B-6 and B-12 have relatively small effects. ${ }^{15}$ Aspirin is the most widely used and least expensive antiplatelet agent.

The choices of statin and of the categories and doses of blood pressure lowering drugs were
Editorial by Rodgers

Department of Environmental and Preventive Medicine, Wolfson

Institute of
Preventive

Medicine, Barts and the London, Queen Mary's School of Medicine and

Dentistry, University

of London, London EC1M 6BQ

NJ Wald

professor

M R Law

professor

Correspondence to: NJ Wald n.j.wald@qmul.ac.uk

bmj.com 2003;326:1419

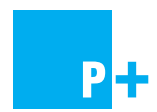

Further tables appear on bmj.com 
Table 1 Effects of the Polypill on the risks of ischaemic heart disease (IHD) and stroke after two years of treatment at age 55-64

\begin{tabular}{|c|c|c|c|c|c|}
\hline \multirow[b]{2}{*}{ Risk factor } & \multirow[b]{2}{*}{ Agent } & \multirow[b]{2}{*}{ Reduction in risk factor } & \multicolumn{2}{|c|}{$\%$ reduction in risk $(95 \% \mathrm{Cl})^{*}$} & \multirow[b]{2}{*}{ Source of evidence } \\
\hline & & & IHD event & Stroke & \\
\hline LDL cholesterol & Statint & $\begin{array}{l}1.8 \mathrm{mmol} / \mathrm{l}(70 \mathrm{mg} / \mathrm{dl}) \\
\text { reduction in LDL } \\
\text { cholesterol }\end{array}$ & 61 (51 to 71$)$ & 17 (9 to 25$)$ & Law et al ${ }^{1}$ \\
\hline Blood pressure & $\begin{array}{l}\text { Three classes of drug at } \\
\text { half standard dose }\end{array}$ & $11 \mathrm{~mm} \mathrm{Hg}$ diastolic & 46 (39 to 53 ) & 63 (55 to 70$)$ & Law et $\mathrm{al}^{16}$ \\
\hline Serum homocysteine & Folic acid $(0.8 \mathrm{mg} / \mathrm{day})$ & $3 \mu \mathrm{mol} / \mathrm{l}$ & 16 (11 to 20$)$ & 24 (15 to 33$)$ & Wald et $\mathrm{al}^{9}$ \\
\hline Platelet function & Aspirin (75 mg/day) & Not quantified & 32 (23 to 40$)$ & 16 (7 to 25$)$ & Table $A$ on bmj.com \\
\hline Combined effect & All & & 88 (84 to 91$)$ & 80 (71 to 87$)$ & \\
\hline
\end{tabular}

LDL=low density lipoprotein.

${ }^{*} 95 \%$ confidence intervals include imprecision of the estimates of both the agent reducing the risk factor and the risk factor reduction decreasing risk. †Atorvastatin $10 \mathrm{mg} /$ day, or simvastatin or lovastatin $40 \mathrm{mg} /$ day taken in the evening or $80 \mathrm{mg} /$ day taken in the morning.

determined from the meta-analysis of short term randomised trials in our companion papers. ${ }^{16}$ The dose of folic acid was the minimum needed to ensure the maximum reduction in serum homocysteine..$^{15} 17$ The long term effect of a specified absolute reduction in LDL cholesterol, blood pressure, and homocysteine expressed as the proportional reduction in the incidence of IHD events and stroke was taken from published sources that were based on systematic reviews of cohort studies. ${ }^{191418}$ Cohort studies show long term effects because the observed differences in risk factors between individuals will have existed for decades. ${ }^{2}$ Predictions from the cohort studies on LDL cholesterol and blood pressure in preventing disease have been corroborated by results of randomised trials, and both cohort studies and trials have shown that a specified reduction in blood pressure or serum cholesterol produces a constant proportional reduction in risk that is independent of the initial value of the risk factor. ${ }^{1341114}$ The average age at which cardiovascular event occurred in the studies was around 60-65 years.

Platelet function is difficult to quantify, and there is inadequate evidence on its association with ischaemic heart disease and stroke. We therefore used direct evidence from randomised trials of the effects of aspirin on disease events. Since the necessary information on stroke and adverse effects with low dose aspirin was not available from published meta-analyses, we conducted one. The efficacy of low dose aspirin (50-125 mg/day) is similar to that of higher doses (160-1500 mg/day), ${ }^{8}$ so we analysed only trials of low dose aspirin. We identified trials of $\geq 6$ months' duration from previous meta-analyses, ${ }^{78}$ from Medline (using the search term "aspirin" in all fields and publication type "clinical trial"), and the Cochrane Collaboration and Web of Science databases. This yielded 15 trials: four were in healthy adults, nine in people with a history of IHD, and two in people with atrial fibrillation. We determined the average proportional reduction in IHD events and stroke, and the prevalence and incidence of adverse effects.

We calculated the combined effect of changing the four risk factors (the effect of the Polypill) by multiplying the relative risks associated with each. We calculated the years of life gained without a heart attack or stroke if people without a previous cardiovascular event used the Polypill from age 55. We used a simple Markov model incorporating the probabilities of three factors-a fatal or non-fatal IHD event or stroke (from an analysis of registry data ${ }^{4}$ ), dying from another cause (from 1999 England and Wales mortality data), and remaining alive without a vascular disease event- stratified by sex and age by year. We then estimated the numbers of events during each subsequent year of life for 100 men and 100 women, and compared these numbers with those calculated from otherwise identical groups not taking the Polypill. From this we determined the extra years of event-free life gained by taking the Polypill.

\section{Results}

\section{Efficacy}

Table 1 shows the effects of the individual agents. By use of statins, LDL cholesterol concentration can be reduced by an average of $1.8 \mathrm{mmol} / \mathrm{l}$. Atorvastatin $10 \mathrm{mg}$ taken at any time of day or simvastatin (or lovastatin) $40 \mathrm{mg}$ taken in the evening or $80 \mathrm{mg}$ taken in the morning after about two years of treatment can reduce the incidence of IHD events at age 60 by an estimated $61 \% .{ }^{1}$ Higher doses produce little further gain. The overall reduction in stroke from an LDL cholesterol reduction of $1.8 \mathrm{mmol} / \mathrm{l}$ is about $17 \%$. This estimate takes account of the reduction in risk of stroke in people with and without existing vascular disease $(35 \% v 11 \%$, a difference which arises because a stroke in people with vascular disease is more likely to be thromboembolic, and statins prevent thromboembolic, but not haemorrhagic, stroke) and also takes account of the proportion of first strokes that occur in people with known vascular disease $(25 \%)^{1}(25 \%$ of $35 \%$ plus $75 \%$ of $11 \%$ making $17 \%$ ).

The five main categories of blood pressure lowering drugs (thiazide, $\beta$ blockers, ACE inhibitors, angiotensin II receptor antagonists, and calcium channel blockers), and the individual drugs within the categories, produce similar reductions in blood pressure, given dose as a ratio of standard dose. ${ }^{16} \mathrm{~A}$ combination of three drugs from different categories in low dose has greater efficacy and fewer adverse effects than using one or two drugs in standard dose. ${ }^{16}$ The blood pressure reduction with three drugs in combination at half standard dose is about $11 \mathrm{~mm} \mathrm{Hg}$ diastolic, reducing the incidence of IHD events by $46 \%$ and stroke by $63 \% .^{16}{ }^{17}$

The maximum effect of folic acid, achieved at a dose of about $0.8 \mathrm{mg} /$ day, ${ }^{15}{ }^{18}$ lowers serum homocysteine by $3 \mu \mathrm{mol} / \mathrm{l}$ (about 25\%) and reduces IHD events by about $16 \%$ and stroke by $24 \%{ }^{9}$

Figure 1 shows our meta-analysis of the 15 randomised trials of low dose aspirin (50-125 mg/day). IHD events were reduced by $32 \%$ and strokes by $16 \%$ (details in web table A). As with statins, aspirin may have a larger preventive effect in people with occlusive 
Trial (First author)

Relative risk $(95 \% \mathrm{CI})$

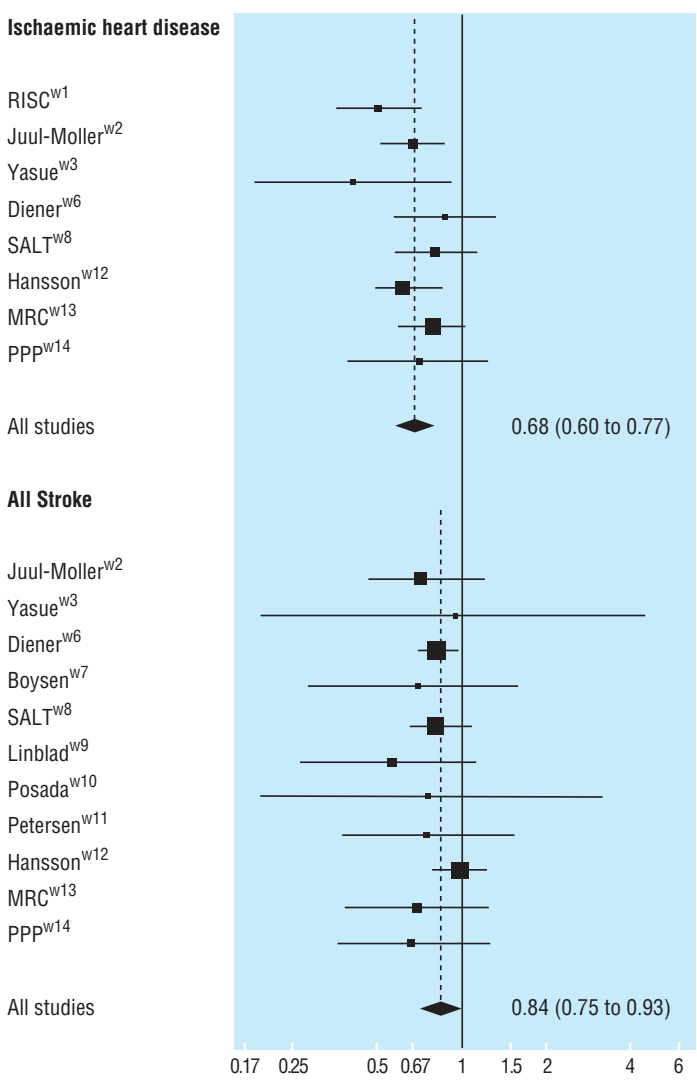

Fig 1 Relative risks (95\% confidence intervals) of ischaemic heart disease events and all strokes (fatal and non-fatal) in 15 randomised trials of low dose aspirin

vascular disease than in those without such disease; the three trials of people without disease indicate a non-significant $9 \%$ reduction in risk of stroke (web table A).

Table 1 shows that changing all four risk factors together reduces the risk of IHD events by $88 \%$ and stroke by $80 \%$. These results are obtained from the product of the relative risk estimates relating to interventions on each risk factor, which is the complement of the proportion of events prevented; thus, preventing, say, $61 \%$ is equivalent to a relative risk of 0.39 . The following example illustrates the calculation. The relative risks of an IHD event for the four interventions in table 1 are $0.39,0.54,0.84$, and 0.66 , the product of which yields a combined relative risk of 0.12 or an $88 \%$ preventive effect (if 100 people who would have had IHD events without intervention were treated, statins would prevent 61 of the 100 events, leaving 39; $46 \%$ of these would be prevented with blood pressure lowering drugs, leaving $21 ; 16 \%$ of these would be prevented with folic acid, leaving 18; and $34 \%$ of these would be prevented with aspirin, leaving $12 ; 88 \%$ have thus been prevented). Reducing one risk factor has a similar proportional effect on risk irrespective of the level of other risk factors, as confirmed by cohort studies and randomised trials. ${ }^{19-22}$ For example, trials of LDL cholesterol reduction show similar proportional reductions in risk in people with high and low blood pressure and in people taking and not taking aspirin. ${ }^{20} 21$

Other than the statin (in respect of IHD), omitting a single component has a relatively minor impact on the combined effect of the residual components, illustrating the robustness of the Polypill concept. Compared with the reductions in IHD events and stroke of $88 \%$ and $80 \%$ respectively with all six components, the reductions were $86 \%$ and $74 \%$ without folic acid, $85 \%$ and $73 \%$ without one blood pressure lowering drug (two instead of three), and $83 \%$ and $77 \%$ without aspirin. So, for example, aspirin prevents $32 \%$ of IHD events when used alone but prevents only an additional $5 \%$ of the original number of expected events when added to the other components in the combination.

Table 2 shows the expected proportion of people who would avoid an IHD event or stroke by taking the Polypill from age 55 and, in those, the average number of event-free life years gained. The estimates take account of deaths from causes other than IHD and stroke. About a third of people taking the Polypill would benefit. On average each will gain 11-12 years of life free from a heart attack or stroke. The gain in life is substantial at all ages.

\section{Adverse effects}

Table 3 summarises the extracranial adverse effects of low dose aspirin from our meta-analysis of 15 randomised trials. Table 4 uses these data together with those published in our companion papers ${ }^{16}$ to show the proportions of people reporting symptoms attributable to any of the components of the Polypill (percentage with symptoms in treated groups minus percentage in placebo groups in trials). If we included the three classes of blood pressure lowering drugs with the lowest prevalence of adverse effects (thiazide, angiotensin II receptor antagonist, and calcium channel blocker ${ }^{16}$ ) in a Polypill formulation, 8\% would be expected to have symptoms attributable to one or more of the six components of the pill, mostly due to aspirin. If we used the three least expensive blood pressure lowering drugs (a thiazide, a $\beta$ blocker, and an ACE inhibitor) instead, a Polypill including these would cause symptoms in about $15 \%$ of people taking the pill.

Of all the components, aspirin has the most serious adverse effects, mainly due to haemorrhage (table B on bmj.com). In our meta-analysis of the trials of low dose aspirin the increase in haemorrhagic stroke (table A on bmj.com) was exceeded by the reduction in thrombotic strokes, producing an overall $16 \%$ reduction in stroke. There was no excess risk of fatal extracranial haemor-

Table 2 Expected benefits in 100 men and 100 women without a known vascular disease who start taking the Polypill at age 55. Calculations are based on a Markov model and allow for other causes of death

\begin{tabular}{lcccccc} 
& \multicolumn{2}{c}{ Men } & & \multicolumn{2}{c}{ Women } \\
\cline { 2 - 3 } \cline { 5 - 6 } Age (years) & No who benefit & Years gainedt & & No who benefit ${ }^{\star}$ & Years gainedt \\
\hline Up to 65 & 7 & 21 & & 3 & 24 \\
\hline Up to 75 & 18 & 16 & & 11 & 18 \\
\hline Up to 85 & 30 & 13 & & 24 & 14 \\
\hline Up to any age & 36 & 12 & & 35 & 11 \\
\hline
\end{tabular}

*Ischaemic heart disease event or stroke avoided or delayed.

†Average years of life gained without an IHD event or stroke in those who benefit. 
Table 3 Extracranial adverse effects of low dose aspirin (50-125 mg) from the meta-analysis of 15 randomised trials

\begin{tabular}{|c|c|c|c|c|}
\hline \multirow[b]{2}{*}{ Adverse event } & \multicolumn{2}{|c|}{ No of events* } & \multicolumn{2}{|c|}{ Excess risk (treated minus placebo) $(95 \% \mathrm{Cl})$} \\
\hline & Aspirin group & Placebo group & As prevalence per 100 people (\%) & As incidence per 1000 person years \\
\hline \multicolumn{5}{|l|}{ Extracranial haemorrhage: } \\
\hline Fatal bleed & 13 & 15 & $-0.01(-0.07$ to 0.05$)$ & $-0.03(-0.20$ to 0.14$)$ \\
\hline $\begin{array}{l}\text { Non-fatal major bleed (required } \\
\text { transfusion or surgery) }\end{array}$ & 51 & 28 & $0.4(0.1$ to 0.6$)$ & $1.2(0.3$ to 2.0$)$ \\
\hline Haematemesis or melaena & 199 & 98 & $0.6(0.4$ to 0.8$)$ & $1.6(1.1$ to 2.2$)$ \\
\hline Any bleed & 1049 & 710 & $2.3(1.7$ to 2.8$)$ & $7.0(5.5$ to 8.6$)$ \\
\hline $\begin{array}{l}\text { Upper abdominal discomfort, } \\
\text { including heartburn }\end{array}$ & 689 & 621 & $1.6(0.0$ to 3.2$)$ & $8.1(0.7$ to 15.4$)$ \\
\hline $\begin{array}{l}\text { Any symptom (any bleed and } \\
\text { abdominal discomfort) }\end{array}$ & 1738 & 1331 & $3.9(2.2$ to 5.6$)$ & $8.6(1.1$ to 16.1$)$ \\
\hline $\begin{array}{l}\text { Adverse effects sufficient to stop } \\
\text { taking the tablets }\end{array}$ & 482 & 438 & $1.6(0.7$ to 2.5$)$ & $5.5(2.4$ to 8.5$)$ \\
\hline
\end{tabular}

*Numbers of participants in the aspirin and placebo groups of each trial were almost identical (see web table B).

rhage, with 13 and 15 deaths in the aspirin and placebo groups respectively in about 17000 people in each (table B on bmj.com), and an excess risk of major nonfatal extracranial haemorrhage (mainly gastric) of 1.2 per 1000 person years (see table 3 ).

\section{Discussion}

The Polypill strategy, based on a single daily pill containing six components as specified, would prevent $88 \%$ of heart attacks and $80 \%$ of strokes. About 1 in 3 people would directly benefit, each on average gaining 11-12 years of life without a heart attack or stroke (20 years in those aged 55-64).

We are confident that the estimated effect is accurate. There is substantial evidence on the individual components of the Polypill, both for risk factor reduction and disease reduction. Extensive evidence exists that reducing the four risk factors by any means lowers the risk of cardiovascular disease. The consistency between evidence from observational studies and trials is persuasive. The estimates of efficacy are robust to imprecision in the separate estimates of the effect of the individual components because overestimates will tend to cancel underestimates. Even if each estimate of the effect of reduction in risk factors on reducing IHD events were $10 \%$ too high (so that in table $1,61 \%$ became $55 \%$, etc) the combined preventive effect of $88 \%$ would only be reduced to $84 \%$.

The percentage reduction in stroke will be greater for non-fatal than fatal events (about $82 \%$ and about $75 \%$, respectively) because statins and aspirin have different effects on thrombotic and haemorrhagic stroke and haemorrhagic strokes are more often fatal.

Table 4 Prevalence of participants in randomised trials reporting symptoms attributable to the Polypill components (in doses specified in table 1)

$\%$ of participants with symptoms*

\begin{tabular}{lcc}
\cline { 2 - 3 } Drug or vitamin & Any symptoms & $\begin{array}{c}\text { Symptoms sufficient to stop } \\
\text { treatment in short term trials }\end{array}$ \\
\hline Statin $^{1}$ & 0.1 & $<0.1$ \\
\hline Thiazide $^{16}$ & 2.0 & 0.1 \\
\hline Angiotensin II receptor antagonist $^{15}$ & $<0.1$ & $<0.1$ \\
\hline Calcium channel blocker $^{16}$ & 1.6 & 0.1 \\
\hline Folic acid $^{2}$ & $<<0.1$ & $<<0.1$ \\
\hline Aspirin (see table 3) & 3.9 & 1.6 \\
\hline
\end{tabular}

*Percentage in treated group minus percentage in placebo group.

\section{Who should take the Polypill}

In people with a previous heart attack or a stroke, without any treatment, cardiovascular disease mortality is about 5\% per year for life. ${ }^{23}$ About half of all cardiovascular deaths occur in individuals with a previous myocardial infarction or cerebral thrombosis. All such individuals should be offered treatment to reduce the reversible risk factors and would benefit from the Polypill. Patients with angina pectoris, transient ischaemic attacks, peripheral arterial disease, and diabetes mellitus should also consider taking the Polypill.

Among people without existing disease, the most discriminatory screening factor is age. As $96 \%$ of deaths from ischaemic heart disease or stroke occur in people aged 55 and over, treating everyone in this group would prevent nearly all such deaths. Using different age cut-offs for men and women, or smokers and non-smokers, or combining several risk factor values with age and sex to produce individual estimates of overall risk would add little discrimination and would probably not justify the added complexity and cost. Figure 2 illustrates this with serum cholesterol, blood pressure, and serum homocysteine. These factors, though aetiologically important, are poor predictors of future cardiovascular disease events. ${ }^{12425}$ There is little separation between the distributions of the risk factors in people who over a specified period do or do not have a disease event. With such closely overlapping distributions there are no cut-off levels that include most people who will have disease events but few of those who will not have them. Cut-off levels that identify the $5 \%$ of the unaffected population who have the most extreme values of the risk factors identify only about $15 \%$ of the disease events (24\% for blood pressure and stroke). The screening performance of cardiovascular risk factors in combination is little better. ${ }^{22}$

The best approach is therefore to treat people with known occlusive vascular disease and everyone aged about 55 or over. There is no need to measure the four risk factors before starting treatment, because intervention is effective whatever the initial levels of the risk factors, ${ }^{11}$ nor to monitor the effect of the treatment, because fluctuations within individuals tend to mask variations between individuals in the systematic effects of the interventions. 


\section{Adverse effects}

The Polypill may not be suitable for some people. $\beta$ blockers are unsuitable for people with asthma, and some people are intolerant of aspirin. Monitoring to prevent rare serious adverse effects of treatment might be considered, measuring serum creatine kinase and transaminase (for rhabdomolysis and hepatitis caused by statins) and serum potassium and creatinine (for acute renal failure caused by ACE inhibitors and angiotensin II receptor antagonists). However, the value of such monitoring is uncertain. The complications are rare, it is not known whether monitoring will avoid them, and the tests lack specificity, so the
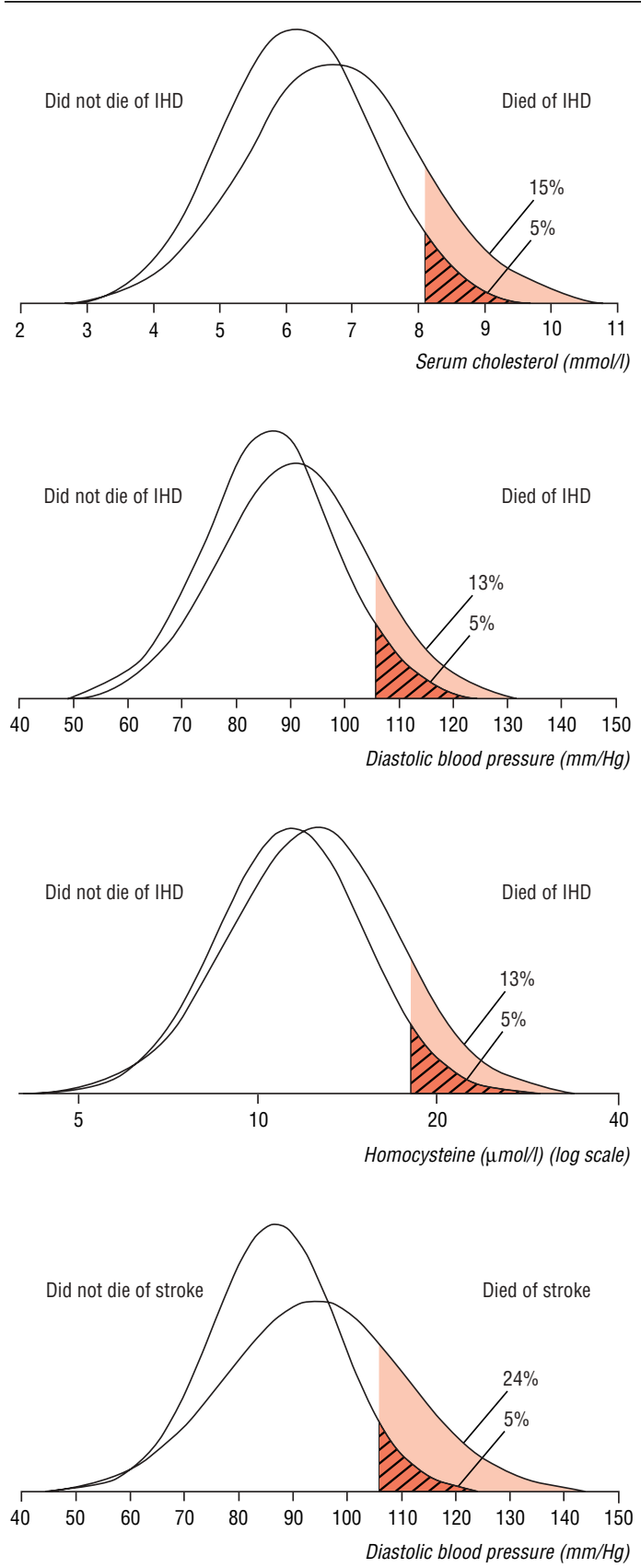

Fig 2 Relative distributions of risk factors in men who subsequently died of ischaemic heart disease or stroke and in men who did not. Gaussian distribution fitted to data from a cohort of 22000 men followed prospectively for 10 years (the BUPA study) 2225

\section{What is already known on this topic}

Four risk factors (LDL cholesterol, blood pressure, homocysteine, and platelet function) that can be reduced by drugs or vitamins account for most cardiovascular disease

Apart from aspirin, the use of such agents has focused on people with high levels of the risk factor

\section{What this study adds}

Intervening on all four risk factors reduces heart attacks and strokes by over $80 \%$

To achieve this large effect in a population requires a combination treatment taken by everyone above a specified age (say 55) and younger people with a clinical history of occlusive arterial disease

A combination pill containing six active components could be widely used

Each component has been used in medical practice for more than 10 years with substantial evidence on safety and efficacy

increased risk of cardiovascular disease after stopping the drug in people positive on monitoring may outweigh any benefit.

\section{Cost and acceptability}

A low cost Polypill could use generic components that are not subject to patent protection (simvastatin (from mid-2003), hydrochlorothiazide, atenolol, enalapril, folic acid, and aspirin). This formulation does not have the lowest rate of adverse effects, but even if about $10 \%$ of people were intolerant of the formulation it would still have considerable public health merit. Those found to be intolerant could be prescribed alternatives to avoid the side effects. Controlled trials of different formulations of the Polypill would provide direct estimates of acceptability.

\section{Conclusions}

The preventive strategy outlined is radical. But a formulation that prevented all cancer and was safe would undoubtedly be widely used, and one that prevented more than $80 \%$ of cardiovascular disease would be even more important, because such deaths are more common than cancer deaths. It is time to discard the view that risk factors need to be measured and treated individually if found to be "abnormal." Instead it should be recognised that in Western society the risk factors are high in us all, so everyone is at risk; that the diseases they cause are common and often fatal; and that there is much to gain and little to lose by the widespread use of these drugs. No other preventive method would have so great an impact on public health in the Western world.

We thank Joan Morris and Alicja Rudnicka for statistical help, and Leo Kinlen, Jeffrey Aronson, Mark Caulfield, David Collier, James Haddow, and Frank Speizer for their helpful comments on drafts of this paper. This paper is based on a lecture given by Nicholas Wald on 18 September 2000 at a meeting in Israel of 
the Israel National Institute for Health Services Policy and Health Services Research.

Contributors: The paper was written by NW and ML. NW generated the idea for the Polypill, which was developed jointly with ML. NW is guarantor.

Funding: None.

Competing interests: The authors have filed a patent application on the formulation of the combined pill described here (application Nos GB 0100548.7 and GB 008791.6, priority date 10 April $2000)$ and a trademark application for the name Polypill.

1 Law MR, Wald NJ, Rudnicka A. Quantifying effect of statins on low density lipoprotein cholesterol, ischaemic heart disease, and stroke: systematic review and meta-analysis. BMJ 2003;326:1423-7.

2 MacMahon S, Peto R, Cutler J, Collins R, Sorlie P, Neaton J, et al. Blood pressure, stroke and coronary heart disease. Part 1:prolonged differences in blood pressure: prospective observational studies corrected for the regression dilution bias. Lancet 1990;335:765-74

3 Collins R, Peto R, MacMahon S, Hebert P, Fiebach NH, Eberlein KA, et al. Blood pressure, stroke and coronary heart disease. Part 2: short-term reductions in blood pressure: overview of randomised drug trials in their reductions in blood pressure: overview of random

4 Law MR, Wald NJ, Morris JK. Lowering blood pressure to prevent myocardial infarction and stroke: a new prevention strategy. Health Technol Assess (in press).

5 PATS Collaborating Group. Post-stroke antihypertensive treatment study. Chin Med J 1995;108:710-17.

6 PROGRESS Collaborative Group. Randomised trial of a perindoprilbased blood-pressure-lowering regimen among 6105 individuals with previous stroke or transient ischaemic attack. Lancet 2001;358:1033-41.

7 Antiplatelet Trialists' Collaboration. Collaborative overview of randomised trials of antiplatelet therapy. I: Prevention of death, myocardial infarction, and stroke by prolonged antiplatelet therapy in various categories of patients. BMJ 1994;308:81-105.

8 Antithrombotic Trialists' Collaboration. Collaborative meta-analysis of randomised trials of antiplatelet therapy for prevention of death, myocardial infarction, and stroke in high risk patients. BMJ 2002;324:71-86.

9 Wald DS, Law M, Morris JK. Homocysteine and cardiovascular disease: evidence on causality from a meta-analysis. BMJ 2002;325:1202-6. (See full version on bmj.com).
10 Schnyder G, Roffi M, Pin R, Flammer Y, Lange H, Eberli FR, et al. Decreased rate of coronary restenosis after lowering of plasma homocysteine levels. N EnglJ Med 2001;345:1593-600.

11 Law MR, Wald NJ. Risk factor thresholds: their existence under scrutiny. BMJ 2002;324:1570-6.

12 Wald NJ, Law MR. Formulation for the prevention of cardiovascular disease. UK patent application No 0008791.6.2000.

13 British Medical Association, Royal Pharmaceutical Society of Great Britain. British national formulary. London: BMA, RPS, 2002. (No 44.)

14 Law MR, Wald NJ, Thompson SG. By how much and how quickly does reduction in serum cholesterol concentration lower risk of ischaemic heart disease? BMJ 1994;308:367-72.

15 Homocysteine Lowering Triallists Collaboration. Lowering blood homocysteine with folic acid based supplements: meta-analysis of randomised trials. BMJ 1998;316:894-8.

16 Law MR, Wald NJ, Morris JK, Jordan R. Value of low dose combination treatment with blood pressure lowering drugs: analysis of 354 randomised trials. BMJ 2003;326:1427-31

17 Wald DS, Bishop L, Wald NJ, Law M, Hennessy E, Weir D, et al. Randomized trial of folic acid supplementation and serum homocysteine levels. Arch Intern Med 2001;161:695-700.

18 Prospective Studies Collaboration. Age-specific relevance of usual blood pressure to vascular mortality: a meta-analysis of individual data for one million adults in 61 prospective studies. Lancet 2002;360:1903-13.

19 ISIS-2 Collaborative Group. Randomised trial of intravenous streptokinase, oral aspirin, both or neither among 17,187 cases of suspected acute nase, oral aspirin, both or neither among 17,187 cases
myocardial infarction: ISIS-2. Lancet 1988;i:349-59.

20 Sacks FM, Pfeffer MA, Moye LA, Rouleau JL, Rutherford JD, Cole TG, et al. The effect of pravastatin on coronary events after myocardial infarction in patients with average cholesterol levels. $N$ Engl J Med 1996;335:1001-9

21 Heart Protection Study Collaborative Group. MRC/BHF heart protection study of cholesterol lowering with simvastatin in 20,536 high-risk individuals: a randomised placebo-controlled trial. Lancet 2002;360:7-22.

22 Wald NJ, Law M, Watt $\mathrm{H}$, Wu T, Bailey A, Johnson M, et al Apolipoproteins and ischaemic heart disease: implications for screening. Lancet 1994:343:75-9.

23 Law MR, Watt HC, Wald NJ. The underlying risk of death after myocardial infarction in the absence of treatment. Arch Intern Med 2002;162:2405-10

24 Wald NJ, Hackshaw AK, Frost CD. When can a risk factor be used as a worthwhile screening test? BMJ 1999;319:1562-5.

25 Screening brief: screening for ischaemic heart disease by serum homocysteine measurement. J Med Screen 2001;8:220. 